Ann. Abeille, 1965,8 (4), 285-297.

\title{
L'EFFET DE QUELQUES FACTEURS SUR LE DÉVELOPPEMENT DU NID A COUVAIN DE L'ABEILLE (APIS MELLIFERA L.)
}

\author{
O. Van LAERE \\ Station d'Entomologie, \\ Section de Recherches apicoles, \\ Wetteren (Belgique)
}

\section{SOMMAIRE}

L'effet des facteurs suivants sur le développement du nid à couvain a été examiné pendant la saison active.

I. La température extérieure - Capacité de la colonie -- Isolation de la muche.

Le développement du couvain ne peut être empêché, en été, par l'instauration artificielle d'une basse température ambiante, qui correspond à la température automnale par laquelle l'activité d'élevage est arrêtée. Le développement du couvain se révèle donc en grande partie indépendant de la température extérieure.

2. L'humidité de l'air.

L'expérience laisse supposer que le développement du nid à couvain peut être influencé par l'humidité de l'air, ce qui, toutefois, devrait être soumis à un examen plus approfondi.

3. L'activité des butineuses - Plantes nectarifères - Sources d'eau disponibles.

Il paraît y avoir une concordance frappante entre le vol total des abeilles et le développement du nid à couvain.

\section{INTRODUC'TION}

L,e développement de la colonie d'abeilles est en rapport direct avec la superficie du nid à couvain ainsi qu'avec l'intensité de la ponte de la reine, quoiqu'un certain pourcentage des cufs ne donne pas naissance à des larves ou à des nymphes. Il en résulte l'importance de l'étude des facteurs qui influent sur l'apparition du couvain dans la ruche. 


\section{MATÉRIEL, ET MÉTHOII:S}

Les observations ont été exécutées sur des colonies normales, installées en plein air, à moins d'autres indications.

Pour les expériences à basse tempêrature $\left(0-20^{\circ} \mathrm{C}\right)$, deux enceintes identiques isolées (isolation thermique au moyen de plaques de polystyrène d'une épaisseur de $10 \mathrm{~cm}$ ) ont été construites en laboratoire. Une des enceintes fut refroidie tandis que l'autre faisait fonction de témoin. Les ruchettes expérimentales furent installées près de la fenêtre de sorte que les abeilles pouvaient sortir en plein air par une issue couverte de toile métallique. La ruchette, avec 3 cadres de $43,5 \mathrm{~cm} \times 28 \mathrm{~cm}$, est représentée sur la figure $\mathrm{I}$.

Les expériences concernant l'humidité de l'air furent exécutées dans un espace de vol climatisé de 6,10 $\mathrm{m}$ de long, $2,20 \mathrm{~m}$ de large et $2,45 \mathrm{~m}$ de haut (fig. 2 ).

Le plafond était en verre mat, au-dessus duquel 37 lampes (PHYTOR I, ACEC) étaient pla cées. L'intensité de lumière à I $\mathrm{m}$ au-dessus du sol était de 800 lux.

\section{PRINCIPAUX RÉSULTATS}

\section{La température extérieure - Capacité de la colonie. Isolation de la ruche}

Nous donnons, tout d'abord, les moyennes des superficies de couvain qui ont été mesurées sur plusieurs colonies durant un an afin d'en comparer l'évolution à la température moyenne pendant la même période (fig. 3). Les abeilles de ces colonies butinaient normalement à l'extérieur.

Ainsi qu'il a été également constaté au cours d'autres années, l'élevage du couvain est arrêté en octobre (le dernier couvain operculé disparaît en novembre) pour reprendre en janvier. I'uisqu'il n'y avait plus de couvain operculé début novembre l'élevage proprement dit fut par conséquent arrêté avant le I5 octobre; on constate également que, vu la présence de couvain operculé le 25 janvier, l'activité d'élevage a donc reprise vers le I $_{5}$ janvier.

Concernant la température ambiante, il est remarquable que l'activité d'élevage de couvain est arrêtée lors d'une température de $12,8^{\circ} \mathrm{C}$ et que, par contre, elle reprend au printemps par une température nettement plus basse, $5,7^{\circ} \mathrm{C}$ pour le cas précité. On n'a pas procédé à des mesures de températures à 1'intérieur des ruches.

On peut se poser la question de savoir quel serait le comportement des abeilles, si une colonie était placée en plein été, artificiellement, dans une température ambiante de $12^{\circ} \mathrm{C}$, tous les autres facteurs étant laissés invariables.

A cette fin, 4 colonies furent transférées en laboratoire le 9 juin et placées deux par deux dans deux enceintes identiques isolées (isolation thermique au moyen de plaques de polystyrène d'une épaisseur de Io $\mathrm{cm}$ ). Les deux enceintes avec les 4 ruchettes (fig. I) furent installées au laboratoire près d'une fenêtre de sorte que les abeilles pouvaient sortir en plein air par une issue couverte de toile métallique. Une de ces enceintes fut refroidie selon la courbe de la figure 4. On n'a pas procédé à des mesures de température à l'intérieur des ruches. 


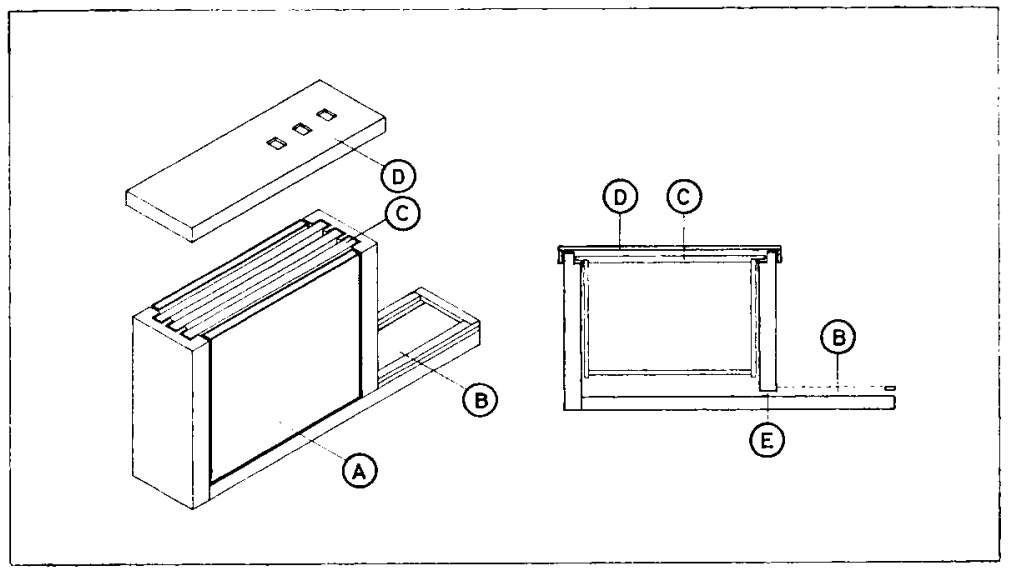

Fici. I. -- Ruchelte expérimenlale

A : paroi latérale en verre isolant (thermopane). B : sortie, couverte de toile métallique. $\mathrm{C}$ : cadre. D : couvercle avec ouvertures pour le nourrissement, contrôle de température... Ii : entrée du corps de ruche.

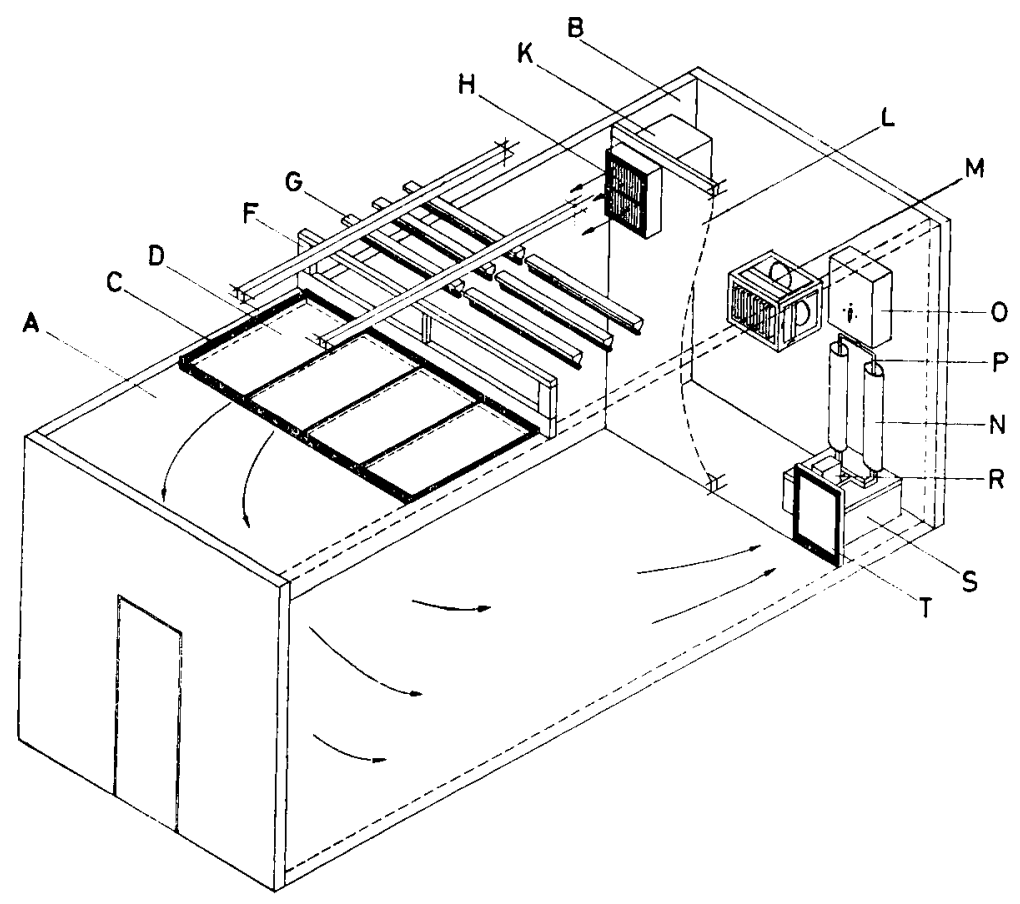

Fl:. 2. - Schéma de l'enceinte climatisée

A : enceinte proprement dite. 3 : endroit pour les appareils. $C$ : cadre métallique. $\mathrm{D}$ : plaque de verre. $F:$ support en bois pour les lampes. (' $:$ lampe. $\mathrm{H}$ : entrée de l'air climatisé. $\mathrm{K}$ : réfrigérateur avec ventilateur incorporé. I. : paroi en plastique. M : ćléments de chauffage et ventilateur central. $\mathrm{N}$ : tubes d'évaporation. $\mathrm{O}$ : ćlément de préchauffage d'eau. $\mathrm{P}$ : conduite d'eau. $\mathrm{R}$ : ventilateur pour l'évaporation d'eau par contre-courant. S : réceptacle pour l'cau superflue. T' ouverture, pourvue de toile métallique. 
La quantité de nourriture a été notée avant et après l'expérience et, compte tenu des quantités administrées, on a constaté les consommations suivantes de nourriture dans la période du 9 au 30 juin (tabl. I). Donc, en moyenne, la consommation de sucre pendant la durée de l'expérience est plus de Ioo p. Ion plus élevée dans les 2 colonies qui ont subi une basse température.

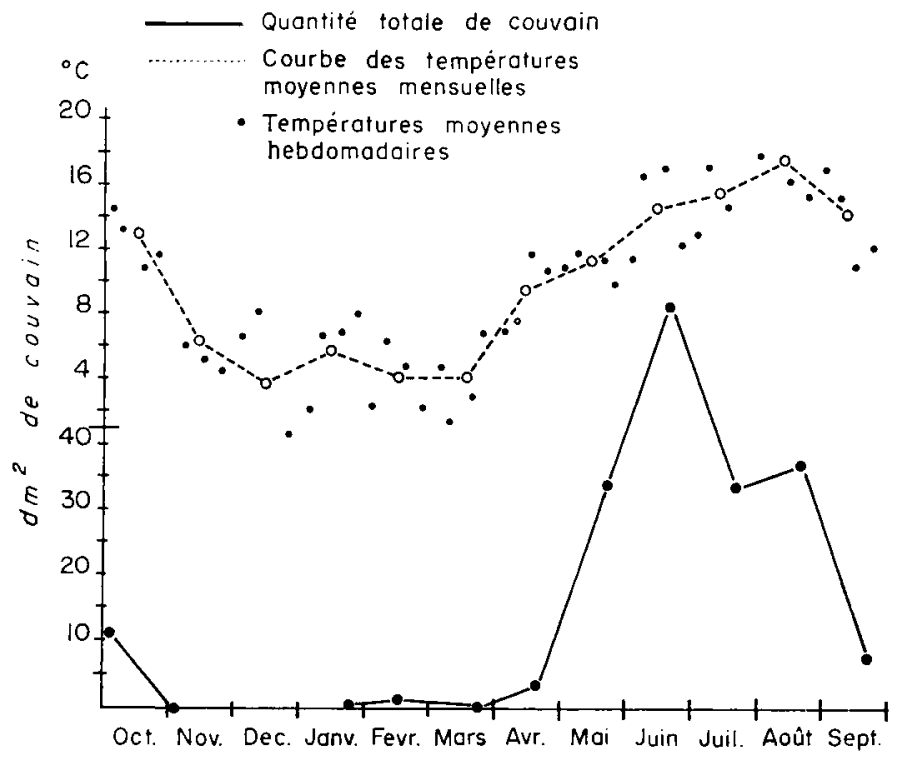

FIG. 3. - Evolution de la superficie de couvain (dm ${ }^{2}$ de superficie de rayon de quatre colonies) comparéc à la courbe de la température extérieure durant la période d'un an

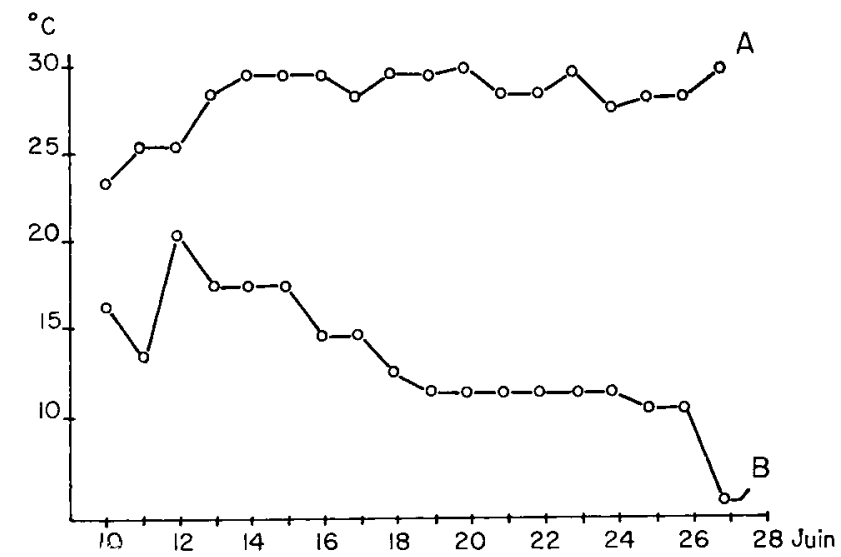

Iig. +. -- Euolution de la température dans les deux enceintes isolées

A : température normale (témoin). B: température dans la partic refroidie.

Le 9 juin, avant le début de l'expérience, des clichés ont été pris afin de mesurer la superficie du nid à couvain et cela dans les 4 colonies. Un second examen a été fait le 30 juin. Les résultats sont donnés dans le tableau 2. 
Les 2 colonies qui avaient subi une basse température ont donc développé en moyenne un nid à couvain de $30,57 \mathrm{~cm}^{2}$, ce qui est encore plus élevé que la superficie du nid à couvain des colonies-témoin $\left(28,6 \mathrm{I} \mathrm{dm}^{2}\right)$.

Le développement du couvain ne peut donc être enrayé en été par l'instauration artificielle d'une basse température automnale par laquelle l'activité d'élevage est arrêtée. Le développement du couvain se révèle donc indépendant pour une grande partie de la température extérieure.

TABLEAU I

Provision et consommation de nourriture dans la période du 9 au 30 juin

Quantité de nourriture en $\mathrm{kg}$ (miel ou solution concentrée de sucre)

\begin{tabular}{|c|c|c|c|c|c|}
\hline No de la ruche & $\begin{array}{l}\text { Quantité } \\
\text { constatée } \\
\text { le } 9 \text { juin }\end{array}$ & $\begin{array}{c}\text { Quantité } \\
\text { administrée } \\
\text { ultérieurement }\end{array}$ & $\begin{array}{l}\text { Quantité } \\
\text { constatée } \\
\text { le } 30 \text { juin }\end{array}$ & \multicolumn{2}{|c|}{$\begin{array}{l}\text { Consommation } \\
\text { totale }\end{array}$} \\
\hline$\left.{ }_{2}^{1}\right\}_{\text {basse température }}$ & $\begin{array}{l}1,1 \\
0,4\end{array}$ & $\begin{array}{l}4 \\
4\end{array}$ & $\begin{array}{l}1,1 \\
1,3\end{array}$ & $\begin{array}{l}4 \\
3,1\end{array}$ & $\begin{array}{r}\text { moyenne } \\
3,55\end{array}$ \\
\hline $\left.\begin{array}{l}3 \\
4\end{array}\right\}$ témoin & $\begin{array}{l}0,6 \\
0,4\end{array}$ & 4 & $\begin{array}{l}3,2 \\
2,5\end{array}$ & $\begin{array}{l}1,4 \\
1,9\end{array}$ & $\begin{array}{r}\text { moyenne } \\
1,65\end{array}$ \\
\hline
\end{tabular}

TABLEAU 2

Mesures de superficies de couvain dans les quatre colonies le 9 et le 30 juin (Méthode photographique planimétrique)

\begin{tabular}{|c|c|c|}
\hline \multirow{2}{*}{ No de la ruche } & \multicolumn{2}{|c|}{ Superficie de couvain en $\mathrm{dm}^{2}$} \\
\hline & le 9 juin & le 30 juin \\
\hline $\left.\begin{array}{l}1 \\
2\end{array}\right\}$ basse température & ${ }_{10,56}^{8,32}$ moyenne : 9,44 & $\begin{array}{l}30,72 \text { moyenne: } 30,57 \\
30,42\end{array}$ \\
\hline$\left.{ }_{4}^{3}\right\}$ témoin & ${ }_{1^{\prime},{ }^{\prime}{ }_{1} 0}^{17,28}$ moyenne : 15,84 & $\begin{array}{l}27,52 \\
29,70\end{array}$ \\
\hline
\end{tabular}

II. L'humidité de l'air

L'humidité est un des facteurs importants qui constituent le climat et l'on peut se demander dans quelle mesure l'humidité de l'air influe sur le développement d'œufs et de larves et si les abeilles règlent l'humidité de l'air de leur nid, lorsque la nécessité s'en fait sentir. 
Afin d'examiner ceci, une colonie d'abeilles a été tenue dans un espace de vol climatisé à une température constante de $26^{\circ} \mathrm{C}$ et des degrés d'humidité relative de 60 p. IOO ( \pm I p. IOO), 68 p. IOO ( \pm I p. IOO), 70 p. IOO ( \pm I p. IOO), 85 p. IOO ( \pm 3 p. IOO) et 88 p. IOO ( \pm 3 p. IOO).

I, a colonie d'abeilles a été placée dans l'espace de vol le 8 juin. On a donné deux fois par semaine du pollen frais et Ioo $\mathrm{cm}^{3}$ de solution sucrée, tandis qu'en même temps on administrait de l'eau sur la ruche même.

La proportion des différents stades du nid à couvain est donnée dans le tableau suivant (tabl. 3 ).

TABLEAU 3

Quantité des stades divers lors du contrôle du nid à couvain

\begin{tabular}{|c|c|c|c|c|c|}
\hline \multirow{2}{*}{ Date } & \multicolumn{4}{|c|}{ Quantité de couvain en dm² } & \multirow{2}{*}{$\begin{array}{c}\text { Humidité } \\
\text { relative } \\
(\%)\end{array}$} \\
\hline & operculé & larves & œufs & total & \\
\hline 6 juillet & - & $\ldots$ & $2 \mathbf{z}^{\prime}$ & 一 & 68 \\
\hline 16 juillet. & ¿alvéoles & 0,6 & 0,8 & $1, t^{\prime}$ & 85 \\
\hline 26 juillet ..... & 1,1 & 0,6 & $0, i$ & 2,1 & 85 \\
\hline 6 août...$\ldots$ & 1,5 & 0,3 & 0,2 & 2,0 & 70 \\
\hline 14 août $\ldots .$. & $1, t_{t}$ & - & $\cdots$ & 1,4 & 60 \\
\hline 27 août ...... & 0,2 & - & 一 & 0,2 & 60 \\
\hline 14 septembre.. & 0,6 & 0,25 & 0,2 & 1,05 & $8 x$ \\
\hline
\end{tabular}

Le 6 juillet, il n'y avait pas de couvain, seulement 24 œufs, plusieurs d'entre eux fraîchement pondus, d'autres de plus d'un jour.

Cette expérience laisse supposer que le développement du nid à couvain peut être influencé par l'humidité de l'air, ce qui toutefois devrait être soumis à un examen plus approfondi.

\section{L'activité des butineuses -- Plantes nectaritères. Sources d'eau disponibles}

La période d'élevage de couvain se situe dans la bonne saison et coïncide plus ou moins avec la période d'activité des butineuses. Comme nous avons constaté une frappante coïncidence entre le moment des premiers vols au printemps et la reprise de l'activité d'élevage du couvain tandis que la température extérieure paraissait de peu d'importance, des observations plus approfondies ont été entreprises afin de connaître le lien entre l'activité des butineuses et le développement du nid à couvain.

Les observations ont été faites depuis le mois de janvier jusqu'à la période de développement maximum du nid à couvain. Dans 4 colonies d'abeilles, la superficie du couvain a été mesurée tous les mois tandis qu'en même temps l'intensité du vol d'abeilles et de la récolte de pollen a été contrôlée.

L'intensité du vol d'abeilles a été constatée en comptant durant 5 minutes entre II heures et midi, tous les 2 jours, le nombre d'abeilles rentrant dans la colonie. En même temps, le pourcentage d'abeilles récoltant du pollen a été noté. 
Lors du contrôle des colonies d'abeilles, la quantité de pollen a été estimée également, tandis que la situation de la provision de miel et le nombre de rayons occupés par les abeilles ont été notés.

Les résultats concernant l'intensité du vol d'abeilles et de la récolte de pollen sont donnés dans un graphique (fig. 5). Chaque colonne verticale indique la moyenne d'un ou plusieurs comptages par io jours. Pour la récolte de pollen, chaque point indique la moyenne des 4 ruches. Lorsqu'il n'y avait pas de vol par suite de matuvais temps, aucune observation n'était faite, ce qui fait que la moyenne précitée ne concerne que les journées de vol. Le nombre de jours de vol par décade est indiqué sur le graphique.

Le 22 mai, la colonie $\mathrm{n}^{0} 4$ étant devenue orpheline fut remplacée. Quelques jours plus tard, la colonie $\mathrm{n}^{0} 3$ reçut une nouvelle reine parce que le nid à couvain montrait des taches irrégulières.

Après les premiers vols en janvier, nous constatons qu'un petit nid à couvain se forme dans 3 des 4 colonies, ce qui se fait quelques jours plus tard dans la $4^{\mathrm{e}}$ colonie (présence de couvain operculé le 16 février (fig. 6)).

Par la suite, il y eut une période de mauvais temps ce qui fait que du début de février à fin mars il n'y eut à vrai dire qu'un seul jour de vol, notamment dans la

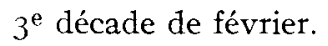

Les mensurations du nid à couvain du 2 I mars nous montrent que dans 2 des 4 colonies, le nid à couvain a complètement disparu tandis que dans les 2 autres il a fortement diminué.

A partir de fin mars des vols réguliers ont lieu et les récoltes de pollen se font régulièrement. En avril et mai, le vol total des abeilles monte constamment ce qui va de pair avec une forte hausse de la capacité du nid à couvain.

Il paraît dès lors y avoir une concordance frappante entre le vol total des abeilles et le développement du nid à couvain (fig. 5 et 6 )).

Notons au passage que la force numérique des colonies au début de 1'année (janvier, février, mars) n'a pas de concordance avec la capacité du nid à couvain, ce qui est démontré dans la figure $6 \mathrm{~B}$ (nombre de rayons occupés). I1 y a toutefois concordance à partir d'avril, ce qui confirme l'expérience pratique que le développement de colonies plus faibles est freiné à partir de mars-avril par une restriction de la superficie du nid à couvain, causée par l'impossibilité pour les abeilles de tenir le couvain à la température requise.

Il est évident que les butineuses ne seront actives que si elles peuvent disposer de suffisamment de plantes nectarifères pas trop éloignées afin de leur permettre de récolter des quantités suffisantes de nectar et de pollen. De même que c'est un fait connu que pour la production de gelée nourricière les abeilles ont un grand besoin d'eau qui doit être à leur disposition en quantité suffisante afin d'arriver à une activité normale. Ces deux facteurs sont dès lors à considérer comme un ensemble par rapport à l'activité des butineuses.

\section{La nourriture.}

SCHAEFER et FARRAR (I946) ont fait des expériences de nourrissement avec 6 fois 3 colonies d'abeilles pourvues de reines sœurs. Un premier groupe de 3 colonies fut nourri au miel. Une $2^{\mathrm{e}}$ colonie reçut du miel et de la farine de soja, tandis que 

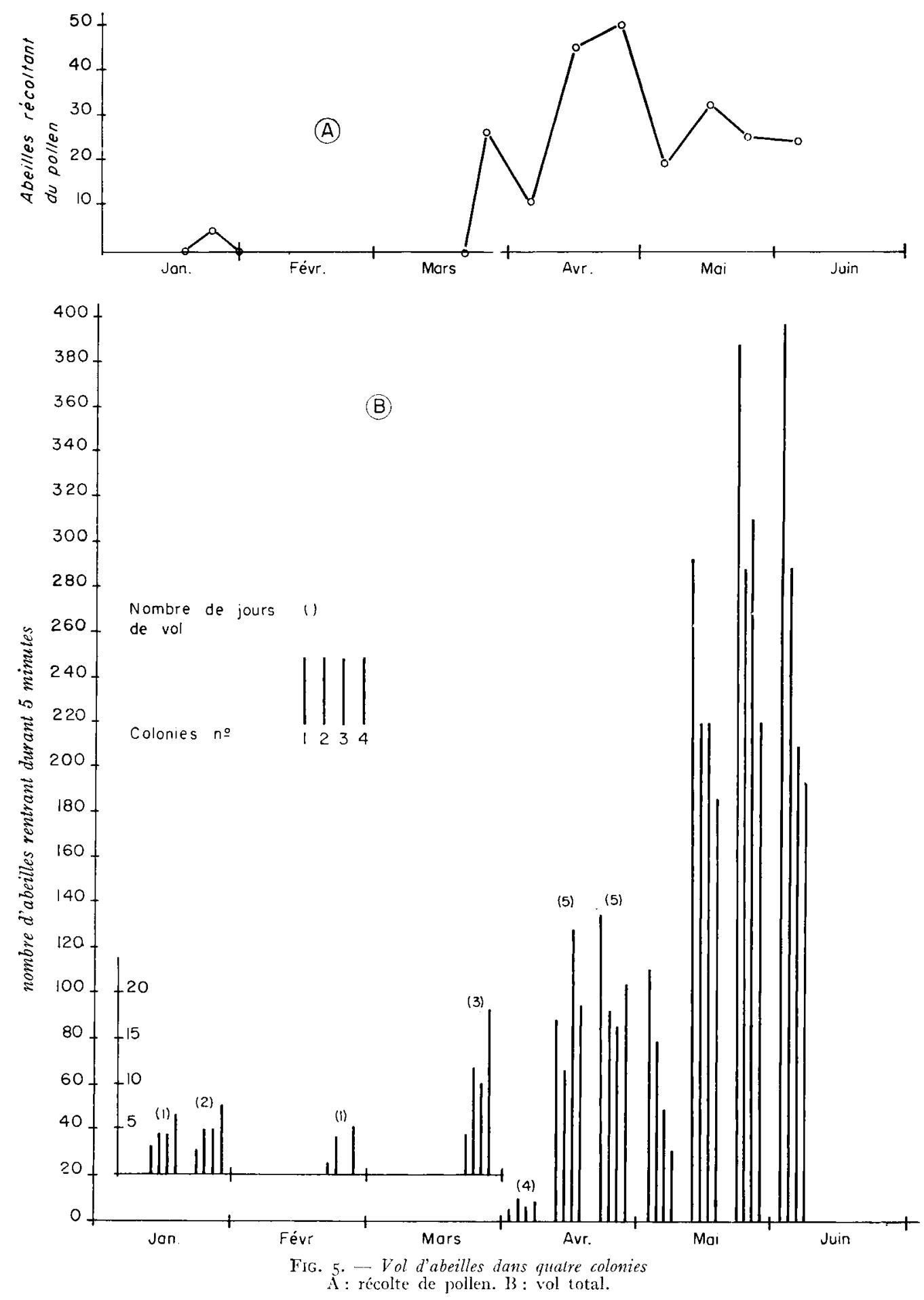


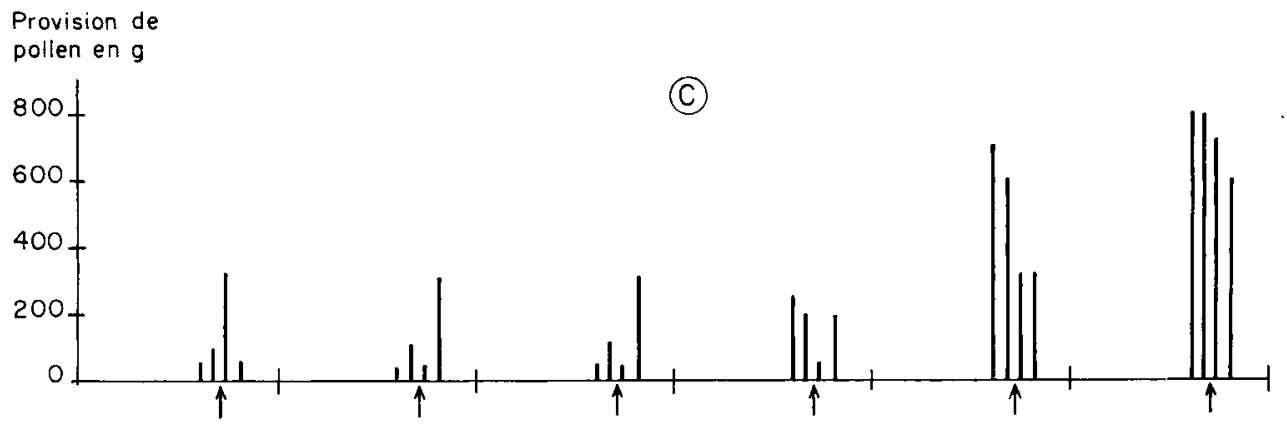

Nombre de

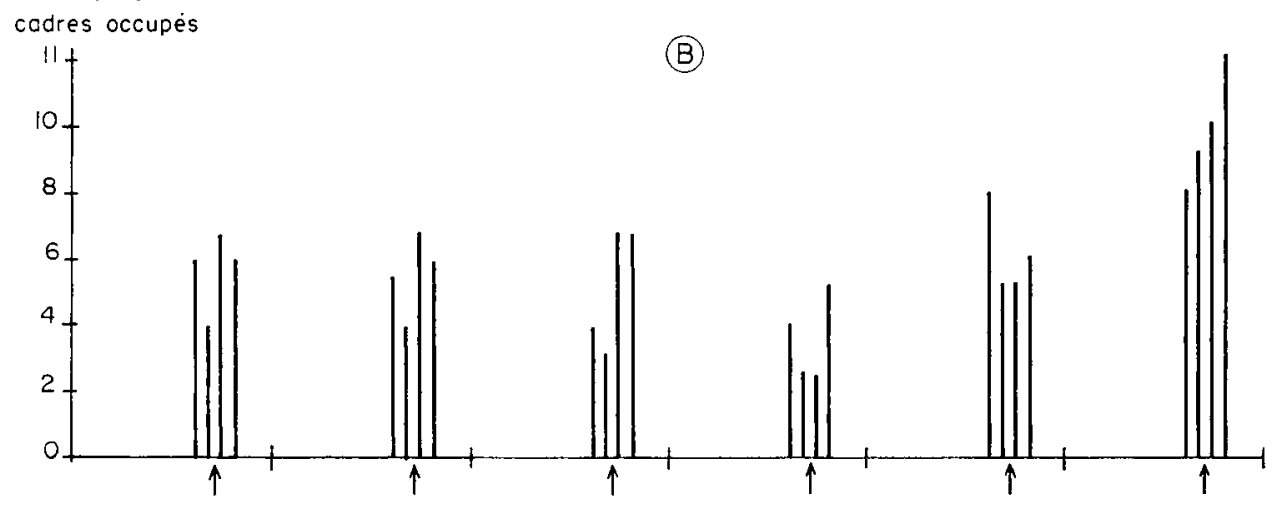

Superficie du nid á couvaín en $\mathrm{dm}^{2}$

(A)

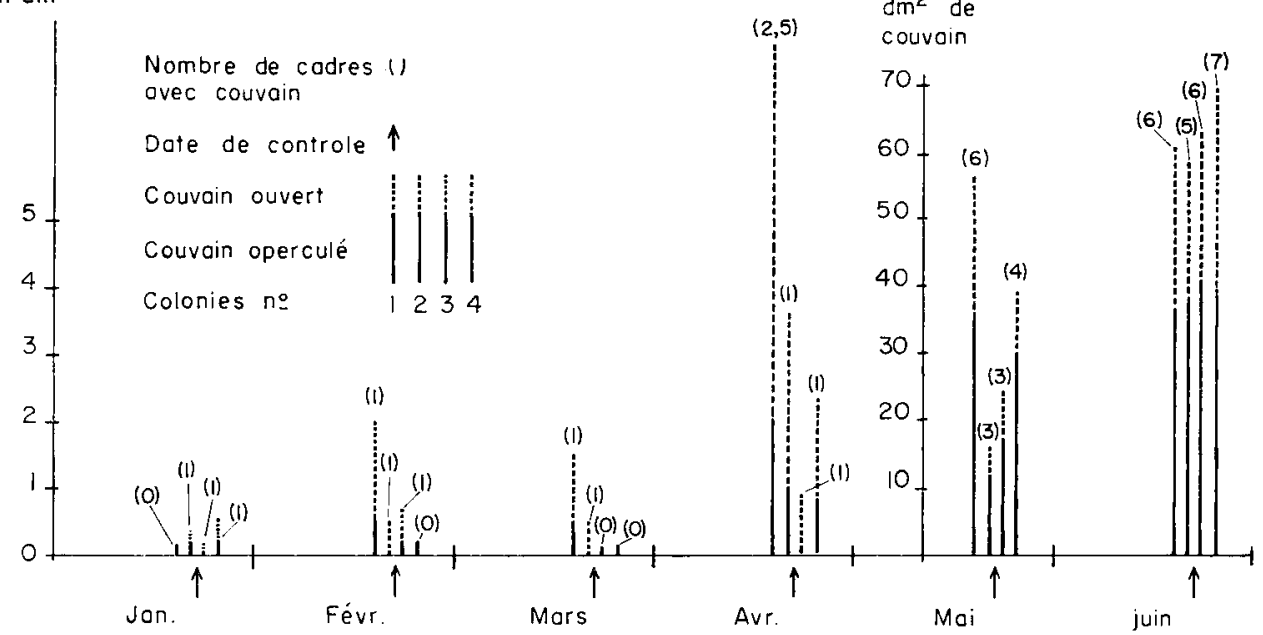

FIG. 6. - Mesures de couvain dans quatre colonies d'abeilles

(A); nombre de rayons occupés (B); réserve de pollen ) C). 
chez d'autres groupes, respectivement I2,5 p. IOO, 25 p. IOO, 50 p. IOO et IOo p roo de la farine de soja fut remplacée par du pollen. Après nourrissement de ces 6 groupes du I2 décembre au 30 janvier, l'on trouve le nombre de cellules à couvain suivant : 575, 2600,4 900, 5 500, 7 300, 8600 , respectivement. A noter qu'à cette période de l'année, dans notre climat, une telle quantité de couvain dans des colonies d'abeilles serait impossible. Ensuite, plusieurs produits pouvant remplacer le pollen furent expérimentés par HAYDAK (I933, I937, I940, I949, I957). Cet auteur employait pour ses expériences de petites colonies d'abeilles composées artificiellement d'environ 7500 jeunes abeilles, n'ayant jamais auparavant consommé de pollen. Ces petites colonies furent nourries au moyen de produits pouvant remplacer le pollen mélangé à du miel jusqu'à obtenir une pâte consistante. Le développement du couvain fut alors enregistré périodiquement. Il a constaté qu'avec un mélange de farine de soja et levure de bière séchée, le nid à couvain était deux fois aussi grand qu'en cas de nourrissement avec un mélange de farine de soja et de pollen de l'année précédente provenant de trappes à pollen et mélangé dans la même proportion.

Avec un nourrissement de pollen frais emmagasiné dans les rayons, la quantité de couvain obtenue était 9 fois plus grande qu'avec le premier mélange de farine de soja et de levure de bière. HAYDAK attribuait cette différence à un manque de protéines nécessaires à la nourriture des abeilles, ce qui l'a poussé à entreprendre plusieurs essais afin d'améliorer la composition de ses nourritures artificielles pour abeilles.

Le meilleur résultat fut obtenu par un mélange de :

3 parts de farine de soja (pourcentage de graisse : 5-7 p. roo) ;

I part de levure de bière séchée;

I part de poudre de lait écréné.

Il conseille d'ajouter à ce mélange Io p. Ioo de jaune d'œuf séché ainsi que Io p. Ioo de caséine finement moulue.

Comme ces 2 produits sont assez chers à employer pratiquement on peut les remplacer par ro p. Ioo de pollen.

Il faut remarquer qu'un nourrissement au pollen ou produits de remplacement n'est nécessaire qu'en période où les abeilles ont un manque évident de pollen (absence de plantes en floraison ou mauvais temps). S'il y a suffisamment de pollen dans les ruches, le produit donné en plus n'est pas accepté. GonTarski (I954) fit des expériences comparatives de nourrissement avec du pollen, de la levure (Waldhof-Höselhefe $\left({ }^{1}\right)$, des extraits de pollen et de levure, ainsi qu'un mélange d'acides aminés provenant de la levure et conclut que tous ces produits sont biologiquement actifs chez des abeilles adultes ; principalement la levure donne un résultat nettement positif.

Dans des expériences faites par Chauvin (I956), l'influence du nourrissement aux hydrates de carbone et aux protéines sur la ponte de la reine durant la bonne saison a été examinée. Les expériences ont été faites avec des petites colonies d'abeilles qui se trouvaient sur un rayon, dont la moitié fut remplacée par une plaque en verre formant ainsi le fond des cellules. Grâce à cette plaque en verre le contenu des cellules est ainsi plus ou moins visible et par conséquent la ponte de la reine peut être suivie. Dans quelques expériences de nourrissement les résultats suivants ont été obtenus.

(') Nom commercial de la préparation de levure comme nourriture d’abeilles. 
r. Lorsqu'on interrompt le nourrissement de solution de sucre durant 3 jours chez des petites colonies la ponte cesse totalement. En effet, des petites colonies ne peuvent pas se suffire à elles-mêmes. Ce résultat confirme un phénomène connu dans la pratique apicole, notamment que le nid à couvain disparaît lorsqu'il y a pénurie de nourriture.

2. Également chez des petites colonies l'on constate environ le double du nombre d'œufs après nourrissement au miel mélangé avec ro p. Ioo de pollen, ceci contrastant avec un nourrissement au miel sans pollen.

L'apport de protéines paraît donc être important, quoique Chavvin (I956) fait remarquer qu'il n'est pas exclu que certaines vitamines puissent jouer un rôle important. Plusieurs auteurs sont d'ailleurs en contradiction sur ce point.

\section{DISCUSSION E'T CONCLUSION}

Concernant la température ambiante, il est remarquable que l'activité d'élevage de couvain soit arrêtée lors d'une température de $12,8^{\circ} \mathrm{C}$ et que, par contre, elle reprend au printemps par une température nettement plus basse, $5,7^{\circ} \mathrm{C}$ pour le cas précité. Nous avons constaté durant d'autres années que ce phénomène est généralement plus prononcé.

Ces observations laissent à supposer que le développement du couvain est dans une certaine mesure indépendant de la température extérieure, ce qui se confirme dans l'expérience avec les ruchettes placées dans un espace où la température était maintenue artificiellement aux environs de I $^{\circ} \mathrm{C}$.

Deux facteurs jouent un rôle essentiel dans la régulation thermique : la force numérique de la colonie et l'isolement de la ruche. Les trois facteurs précités (la température extérieure, la capacité de la colonie et l'isolement de la ruche) forment donc un ensemble par rapport à la superficie du nid à couvain.

En ce qui concerne l'activité des butineuses, la comparaison des résultats sur le graphique de la figure 5 et ceux de la figure 6 s'impose. On peut remarquer facilement une concordance frappante entre le vol total des abeilles et le développement du nid à couvain.

Toutefois, le pourcentage d'abeilles récoltant du pollen baisse à partir d'un moment déterminé (début mai), ce qui donne l'impression qu'une colonie d'abeilles ne récolte que la quantité de pollen nécessaire à l'alimentation du couvain. Mais ceci ne paraît toutefois pas être le cas. En effet, en avril la plupart des plantes en floraison donnent du pollen (Salix, Taraxacum, arbres fruitiers), ce qui fait qu'un pourcentage assez élevé des butineuses s'y concentre. De même que les grandes quantités de pollen que l'on trouve à l'arrière-saison dans des colonies normales n'indiquent pas non plus un rapport entre le nombre d'abeilles récoltant du pollen et le nombre de larves à nourrir.

Comme le démontre la figure 6 , nous remarquons également jusque début avril qu'il n'y a pas de concordance entre la provision de pollen et la quantité de couvain. Plus tard, la provision de pollen augmente, résultat logique de l'augmentation du nombre des abeilles dans les colonies et, par conséquent, d'une plus grande activité de vol. 


\title{
REMERCIEMENTS
}

L'auteur tient à remercier M. le Pr Dr h.c.J. VAN DEN BRANDE pour l'encouragement donné à ces recherches et les facilités offertes dans son laboratoire.

\section{SUMMARY}

\author{
THE INFLUENCE OF SOME FACTORS \\ ON THE BROOD-NEST DEVELOPMENT ("APIS MELLIFICA $")$
}

The influence of the following factors on the brood-nest dévelopment has been studied during the active season :

\section{External temperature - Capacity of the colony - Isolation of the hive.}

The brood development cannot be prevented by establishing artificially a low environmental temperature, being the mean temperature at which breeding activity in autumn stops. Thus the development of the brood is greatly independant of the environmental temperature.

\section{Air humidity.}

The experiment is carried out in an air-conditioned flight room. The brood-nest development seems to be influenced by air humidity, although no definite conclusions could be drawn from the present investigations.

3. Foraging activity of the bees - Nectar plants - Water sources.

The experiments show a striking confornity between the total flight of the bees and the deve. lopment of the brood-nest.

\section{RÉFÉRENCES BIBLIOGRAPHIQUES}

Allex M. D., JefFree E. P., I 956. The influence of stored pollen and of colony size on the brood rearing of honeybees. Ann. appl. Biol., 44, 649-656.

BACK E., 1956. Einfluss der im Pollen enthaltenen Vitamine auf Jebensdauer, Ausbildung der Pharynxdrüsen und Brutfähigkeit der Honigbiene. Insectes socianx, 3, 285-292.

Bodenheimer F. S., Ben-Nerya A., I 937. One-year studies on the biology of the Honeybee in Palestine. Ann. Appl. Biol., 24, 385 403.

Chauvin R., I956. Les facteurs qui gouvernent la ponte chez la reine des abeilles. Insectes sociaux, 3 499-504.

DE GRoOT A. P., 1953. Protein and amino acid requirements of the honeybee (Apis mellifica I.). Proetschritt Univ. Utrecht, $90 \mathrm{p}$.

Gontarski II., I 954. Untersuchungen über die Verwertung von Pollen und IIefe zur Brutpflege der Honigbiene. Z. Bienenforsch., 5, I-20.

HAYdaK M. H., I933. Der Nährwert von Pollentrsatzstoffen bei Bienen. Arch. Bienenkunde, 14, 185-219. HAYDAK M. H., I 937. Changes in weight and nitrogen content of adult worker bees on a protein-free diet. J. agric. Res., 45, $79 \mathrm{I}$.

HAYDAK M. II., 1949. The Queen Honeybee. State apiarist report. Iowa.

HAYdaK M. H., I957. Is there a pollen substitute equal to pollen? Amer. Bee J., 97, 90-91.

HAYdaK M. H., 1958. Wintering of Bees in Minnesota. J. econ. Entomol, 51, 332-334.

Hess W. R., 1925. Die Temperaturregulierung im Bienenvolk. Z. i'ergleich. Physiol., 4, 465-487. 
Hitchcock J. D., I956. Honeybee queens whose eggrs all fail to hatch. J. econ. Eniomol., 49, 1 I-44. LAVIE P., I954. L'enregistrement thermique continu dans les populations d'Apis mellifica au cours de l'hivernage. Insectes sociaux, 1, 39-48.

Levin M. D., Nye W. P., KNowlton G. K., I95I. Feeding pollen supplement and pollen substitule to honeybees.

Lindauer M., I954. Temperaturregulierung und Wasserhaushalt im Bienenstaat. Z. vergleich. Physiol., 36, $39 \mathrm{I}-43^{2}$.

Nolan W. J., 1925. The brood-rearing cycle of the Honeybee. U. S. Dept. Agric. Bull., no 1349,55 p.

RENNER M., I955. U̇ber die Haltung von Bienen in geschlossenen künstlich beleuchteten Raümen. Naturwissenschaften, 39, 539-540.

Svoboda J., I940. Über den Wert des Pollens als Nahrungsmittel für Bienen. Schweiz. Bienenzgt., 63, 206-209.

VAN LAERe O, VAN DEN BRANDE J., I958. Observations sur la diapause de reproduction chez la reine d'abeilles, C.R. XVII e Congr. Apic., ,20-423. 